\title{
DIE KÖNIGLICH ZOOLOGISCHE GESELLSCHAFT „NATURA ARTIS MAGISTRA" UND DIE TIERPSYCHOLOGIE
}

\author{
voN \\ J. A. BIERENS DE HAAN
}

\section{EINLEITUNG}

Die Königlich Zoologische Gesellschaft „Natura Artis Magistra”, deren Hundertjahrfeier wir diesen Sommer feiern, hat sich von Anfang an die Aufgabe gestellt, mehr als die Eigentümerin eines Gartens zu sein, in welchem mehr oder weniger seltene und kuriose Tiere für das Publikum zur Schau gestellt sind, und hat immer danach gestrebt, ein kulturelles und wissenschaftliches Zentrum in der Stadt ihrer Gründung darzustellen. Das Glück wahr ihr dabei hold: gelegen in der Mitte einer grossen, aber nicht zu grossen Stadt, gross genug, als Reichshaupt- und Universitätsstadt ein Kultur-Mittelpunkt des Landes zu sein, und andererseits doch wieder nicht so gross, dass dabei die einzelnen Institute im Ganzen verlorengehen, vermochte sie sich je länger je mehr mit Erfolg zu einem solchen kulturellen und gesellschaftlichen Zentrum zu entwickeln. Besonders seitdem 1877 das städtische Athenaeum zu einer Universität ausgebaut wurde, ist die Königlich Zoologische Gesellschaft durch enge Bande mit der universitären Zoologie verbunden: in der Mitte ihres Gartens liegt das Zoologische Institut der Universität, während daneben ein gemeinschaftlich der Universität und der Gesellschaft gehörendes Zoologisches Museum wissenschaftlich wertvolle Sammlungen enthält und eine an alten und seltenen Büchern reiche Bibliothek den holländischen Zoologen grosse Vorteile darbietet. Und als eine Besonderheit kommt noch hinzu, dass die Gesellschaft seit einiger Zeit auch ein Zentrum für einen speziellen Zweig der zoologischen Wissenschaft, nämlich die Tierpsychologie, bildet.

Es wird einleuchten, dass die Zoologischen Gärten berufen sind, eine wichtige Rolle zu spielen für die Entwicklung der Tierpsychologie. Die Tierpsychologie muss' sich aus Beobachtungen des tierischen Verhaltens; sowohl in freien natürlichen Situationen als im zu einer bestimmten F'ragestellung konstruierten 
Experiment aufbauen ${ }^{1}$ ). Tierpsychologische Beobachtungen in der freien Natur lassen sich jedoch hauptsächlich nur an niederen Tieren ausführen: bekannt sind die klassischen Beobachtungen, die FABRE in den zehn Teilen seiner "Souvenirs entomologiques" festgelegt hat, und die ebenso wichtigen Beobachtungen an Ameisen und Bienen der beiden Hubers, Forels, WheeLERS; $u$ : a. Von den höheren Tieren sind es aber nur die Vögel, die in ihrem Freileben Anlass zu tierpsychologisch wichtigen Beobachtungen gegeben haben; von den amerikanischen Forschern, die Feldstudien an Affen gemacht haben, konnte eigentlich nur CARPENTER ${ }^{2}$ ) durch glücklichè Umstände Beobachtungen an Brüllaffen machen, welche die dafür aufgewandten Anstrengungen lohnten. Zur Ausfüllung dieser Lücke können nun 'Tiergärten mehr oder weniger helfen.

Ich will natürlich nicht behaupten, dass das Tier im Tiergarten völlig dem Tiere in der freien Natur gleichzustellen ist. Das eingesperrte Tier wird durch die beschränkte Bewegungsfreiheit daran gehindert, sich ganz ausleben zu können, und es fehlt ihm der Drang der Not, sich in einer ihm feindlichen Natur behaupten zu müssen, um ganz seine tierische Natur und seine körperlichen und geistigen Eigenschaften zu zeigen. Doch verhindert dies nicht, dass es in seinem beschränkten L_ebensraum dasselbe Tier bleibt wie vorher. Noch oft findet man bei vielen Laien die irrige Meinung, dass das Tier im Tiergarten schnell degeneriere und verblöde, eine Vorstellung, welche wahrscheinlich dadurch entstanden ist, dass der Laie sich das Tier in seinem Freileben als ein immer handelndes Wesen vorstellt. Der Löwe wird durchweg brüllend und beutejagend gedacht und der Adler so, als ob er immer hoch am Firmament seine Kreise ziehe, sodass der Laie, wenn er das Tier in einem nichtaktiven Zustande vorfindet, oft geneigt ist, dies einer Gefangenschaftsverblödung zuzuschreiben. Er übersieht dabei ganz, dass das Tier im Freileben nur dann sich abmüht, wenn es von Hunger gequält, von Geschlechtstrieb beunruhigt oder von einem Feinde bedroht wird, sodass, wenn im Tiergarten diese Faktoren fehlen, es nur das bei den meisten Tieren ziemlich geringe Bedürfnis an Bewegung ist, das die Tiere in Aktivität versetzt.

Dass das Tier auch im Tiergarten sich selbst gieich geblieben ist ${ }^{3}$ ), zeigt

I) Uber die Methode sehe man näher: J. A. Bierens inf HAAN, Die tierpsychologische Forschung. Ihre Ziele und Wege. Leipzig, Joh. Amb. Barth, 1935.

2) C. R. Carpenter. A field study of the behavior and social relations of howling monkeys (Alouatta palliata). Comp. Psychol. Mon., X, 19.34.

3) Ein typisches Beispiel davon, wie das Tier in Gefangenschaft unverändert bleibt, bot mir vor einiger Zeit ein Schweinsaffe, der als junges Tier in den Garten gekommen war, und seitdem immer in einem Käfig gelebt hatte. Es gelang diesem Tier nämlich, zu entwischen und aus einem Fenster des Laboratoriums hinauszuspringen. Sofort flüchtete es dann hinauf in einen hohen Ulmenbaum, kletterte in den Ästen hinauf und hinunter, sprang von einem Baum auf einen anderen, ebenso, wie Affen dies in der freien Natur tun. Erst nach etwa einer. Woche zwang ihn der Hunger, hinunterzukommen. Ungeachtet der Tatsache, dass das Tier sich länger in Gefangenschaft als in der freien Natur aufgehalten hatte, war es direkt wieder ganz ein Wildtier geworden, körperlich und geistig ganz der Situation gewachsen. 
sich schon aus einfachen Beobachtungen, die man in jedem Tiergarten machen kann, sobald das Tier nur eine Ursache hat, sich in irgend einer Beziehung auszuleben. Am meisten fällt uns dies auf, wenn wir ein offenes Auge für die sozialen Beziehungen in dem beschränkten Raume des Käfigs oder des Geheges haben. Der Ententeich, das Affenhaus oder der Affenfelsen, sogar das Bassin in Aquarium geben fortwährend Gelegenheit zu Beobachtungen über die Rolle des Herrschers und der Beherrschten, und über die soziale Struktur einer Tiergemeinschaft, wenn mehrere Tiere zusammen sind. Auch auf dem Gebiete der Paarungshandlungen mit ihrem Vorspiel (Balz), der Versorgung der Nachkommenschaft, des Familienlebens verhalten sich die Tiere im Garten wie in der freien Natur. Und warum auch nicht? Der Tiergarten möge ein zu einem bestimmten $Z$ weck gestaltetes Stück Natur sein, es bleibt dennoch ein Stück Natur, sobald man ihn mit Tieren belebt.

Hinzu kommt dann noch, dass durch dessen einfachen Aufbau die Beobachtung im Zoologischen 'Garten oft viel leichter und genauer. geschehen kann als in der freien Natur. Als der Verfasser im Amsterdammer Zoo die Balz des Argusfasans beobachtete $(\mathrm{I})^{1}$ ) bei einem Tier, das sich nicht im wenigsten dadurch stören liess, dass der Autor in einigen Metern Entfernung zuschaute, konnte er dadurch ohne Mühe Einzelheiten wahrnehmen, die BEEBE ${ }^{2}$ ), der die Balz auf den natürlichen Balzplätzen studierte und von einer Grube aus beobachten musste, in welcher er, von Ameisen gequält, unbeweglich durch das Gebüsch hindurchschauen musste, entgangen sind. Gerade die Tatsache, dass die Tiere im Zoo ganz an Menschen gewöhnt sind und meistens keine Angst für sie haben, so lange sie in einiger Entfernug ausserhalb ihrer Gehege umhergehen, erleichtert die Beobachtung ihres Verhaltens in hohem Masse.

Jedoch kann die Tierpsychologie allein durch Beobachtungen nicht zu einer Wissenschaft auswachsen. Diese müssen vielmehr durch Experimente ergänzt werden. Und auch zu diesen eignet sich die Welt des Tiergartens viel besser als die freie Natur. Die Gründe dazu sind oben schon gegeben: die relative Zahmheit der Tiere, der relativ einfache Aufbau ihrer Welt, machen das Anstellen von einfachen Experimenten viel leichter, als wenn dies im Felde geschehen müsste. Aber daneben soll der Zoologische Garten, wenn er zu einer eigentlichen Forschungsstätte für Tierpsychologie eingerichtet ist, ein - wenn auch einfaches - Laboratorium besitzen, wo die Tiere in Käfigen gehalten und den strengen Bedingungen des Laboratoriumexperimentes unterworfen werden können. Seinerseits kann der Garten die Aufgabe des Laboratoriums erleichtern, indem von ihm die Tiere geliefert und auch verpflegt werden, welches letztere in vielen anderen Laboratorien bisweilen wohl etwas zu wünschen übrig lässt.

Im Zoologischen Garten der Gesellschaft „Natura Artis Magistra” ist nun

1) Die eingeklammerten Zahlen beziehen sich auf die Liste der Arbeiten am Ende dieses Artikels.

2) W. BeEBe. A Monograph of the Pheasants. IV. London, 1922.

Bijdragen tot de Dierkunde, Af. 27 
durch glückliche Umstände der Zustand erreicht, dass einerseits der Garten selbst für tierpsychologische Beobachtungen dienstbar gemacht wird, andererseits seit etwa zwölf Jahren ein kleines Laboratorium vorhanden ist. "Artis" ist wohl der erste Garten, wo man dies erreicht hat. Seit einigen Jahren hat jetzt auch der Zoologische Garten in Münster in Westfalen eine besondere Arbeitsstätte für Tierpsychologie, die unter Leitung von Dr. Werner FischeL steht. Diese fördernde Stellungnahme gegenüber der Tierpsychologie ist der jubilierenden Gesellschaft entschieden zur Ehre anzurechnen, und ich glaube daher, dieses Jubileum als Anlass benutzen zu dürfen, eine kurze Zusammenfassung dessen $z u$ geben, was in dieser Zeit innerhalb des Gartens an tierpsychologischen Beobachtungen und experimentellen Arbeiten verrichtet worden ist.

Vorher noch einige kurze historische Bemerkungen. Der erste, der im Garten tierpsychologische Arbeiten ausführte, war Herr Inspektor A. F. J. Portielje. Seit seiner Anstellung im Garten im Jahre 1906, hat er stets ein offenes Auge für das psychische Moment im Tiere gehabt, und davon oft in populären Schriften und Vorträgen, vor allem in seinem "Artis-Buch" ${ }^{1}$ ) und seinem vor kurzem erschienenen Buche über den tierischen Instinkt ${ }^{2}$ ), Belege gegeben. Unter seiner Leitung haben oft Schüler einer Schule für Montessori-Unterricht, sowie Studenten und Studentinnen der Biologie, tierpsychologische Beobachtungen an Vögeln und Säugetieren ausgeführt. Des Verfassers eigene Bande mit "Artis" datieren vom Anfange des Jahres 1925, als nach der Übersiedlung Prof. Buytendyks von Amsterdam nach Groningen, das Biologische Institut der "Vrije Universiteit" geschlossen wurde. Der damalige Direktor der Zoologischen Gesellschaft, Dr. C. KERBERT, war dann so freundlich, dem Verfasser für seine Untersuchungen ein kleines Treibhaus in der Fasanerie zur Verfügung zu stellen. Dies hatte nur den Nachteil etwas kleiner Dimensionen (bei einer Grundfläche von $3 \times 3^{1 / 2} \mathrm{~m}$ war es nur $2.30 \mathrm{~m}$ hoch, sodass, um einen Affenkäfig zu beherbergen, das Dach des Gebäudes erhöht werden musste!), wodurch allein mit Tieren gearbeitet werden konnte, die sich in dem einen Käfig zusammenbringen liessen. Im Sommer 1928 gab der jetzige Direktor Dr. A. L. J. SuniER dem Verfasser einen grösseren Raum, der aber den Nachteil hatte, schlecht heizbar und dadurch für den Winter weniger geeignet zu sein. Erst als im Herbst ${ }_{1928}$ der Vorstand der Zoologischen Gesellschaft den nicht mehr benutzten Dachboden eines ehemaligen Gebäudes des Reichs-Brieftaubendienstes $z u$ diesem $Z$ weck freimachte, konnte durch Umbau ein einfaches aber praktisch verwendbares Laboratorium eingerichtet werden. Dieses Laboratorium wurde früher schon an anderer Stelle beschrieben ${ }^{3}$ ), sodass wir darauf nicht näher zurückzukommen brauchen.

Wir wollen nummehr versuchen, eine Übersicht von dem zu geben, was aus dem Garten und dem Laboratorium der Kön. Zool. Gesellschaft an tierpsychologischen Forschungsergebnissen hervorgegangen ist. Hierbei wollen wir nur über solche Untersuchungen berichten, die Anlass zu einer wissenschaftlichen Publikation gegeben haben. Da es sich für unseren $Z$ weck nur um empirische, im Garten erworbene Ergebnisse handelt, sind theoretische oder zusammenfassende Arbeiten der in „Artis” arbeitenden Forscher nicht mit besprochen und

I) A. F. J. Portielje und S. Abramsz. Het Artis Boek. I-II. Zutfen,1922.

2) A. F. J. Portielje. Dieren zien en leeren kennen. Amsterdam, 1938.

3) J. A. Bierens de. HAAN. Zoologischer Garten und Tierpsychologie in Amsterdam.

Der Zool. Garten, III, I930. 
auch ihre theoretischen Betrachtungen nur beiläufig erwähnt. Es scheint am geeignetsten, die Arbeiten einerseits nach der Stelle, wo die Untersuchungen ausgeführt sind (Garten, Aquarium, Laboratorium), und andererseits nach der Art dieser Untersuchungen selbst (Beobachtungen, Experimente) zu ordnen. Wir fangen daher mit den Beobachtungen und einfachen Experimenten an, die im Garten der Gesellschaft selbst ausgeführt sind.

\section{IM GARTEN DER GESELLSCHAFT ANGESTELLTE BEOBACH- TUNGEN UND EINFACHE EXPERIMENTE}

Wie gesagt, war Portielje der erste, der den Garten und seine Tiere für tierpsychologische Untersuchungen zu verwenden wusste. Sein besonderes Verdienst war es dabei, dass er der erste in unserem Lande war, der die Bedeutung eines eingehenden Studiums und einer psychologischen Analyse des verwickelten Verhaltens der Vögel, besonders bei der Paarung und Brutpflege, wie sich dies so gut in einem Tiergarten beobachten lässt, für die Tierpsychologie verstanden hat.

Nachdem er (2I) eine biologische und psychologische Beschreibung der Paarungs- und Brutinstinkte von einigen dort lebenden Vögeln, wie dem Riesenreiher Ardea goliath, dem Weissnackenkranich Pseudogeranus leucauchen und dem schwarzen Geier Catharista atrata gegeben und $(22,23)$ einige merkwürdige Instinkte und Gewohnheitsbildungen bei einigen anderen Vögeln beschrieben hatte (so beobachtete er, dass der Kiebitz instinktiv oder auf Erfahrung beruhend, schwingende Bewegungen mit dem nach vorn ausgestreckten Fuss macht, wodurch er die Würmer aus dem Boden hervorjagt, während die Silbermöwe und die Brandgans hierzu trampelnde Bewegungen mit beiden Füssen machen und der Singschwan solche Bewegungen macht, wenn er oder das Weibchen sich mit den Jungen an einer Stelle befindet, wo in den höheren Wasserschichten keine Daphnien zu finden sind, sodass dadurch die Daphnien nach oben geführt werden und von den Jungen verspeist werden können), hat Portielje sich vor allem mit der Psychologie von vier im Garten lebenden und dort brütenden Vögeln, nämlich dem Pampasstrauss Khea americana (24), der Rohrdommel Botaurus stellaris (25), dem Kormoran Phalacrocorax carbo (27) und der Silbermöwe Larus argcntatus (28) beschäftigt, wobei er, soweit es sich um einheimische Tiere handelte, seine Beobachtungen im Garten an Wahrnehmungen in der freien Natur kontrolliert hat.

Beim Pampasstrauss (24) wurde dabei der Balztanz der beiden Tiere beschrieben, bei welchem der Hahn abwechselnd den einen und den anderen Flügel ausbreitet, und den eigentümlichen Balzruf, welchem er seinen Namen „Nandu" verdankt, hören lässt. Später auch noch, wenn schon ein Ei im Neste liegt, nimmt der Hahn eine Prunk- oder Imponierstellung an, worauf eine Paarung folgt. Das Brutgeschäft und die Aufzucht der Jungen werden bei diesem Tier vom Hahn besorgt, der sich aber nur um Eier kümmert, die im 
Neste oder am Nestrand liegen. Wenn das Weibchen im Begriff steht, ein Ei zu legen, nähert es sich dem Nest, wonach der Hahn einen Flügel ausstreckt und darauf das vom Weibchen gelegte Ei auffängt und in das Nest hereinholt, dabei eventuell mit dem Schnabel nachhelfend. Kommt das Ei etwas weiter zu liegen, dann wird dazu der als ein Haken gekrümmte Hals benutzt, wobei eventuell das Weibchen mithilft. In der Ausbreitung der Flügel, die der Hahn bei seiner Balz ausführt, will nun Portiklje eine Scheinbewegung dieses Eiauffangens sehen, die die Funktion hat, das Weibchen in eine sexuelle Erregung zu bringen. Solche Handlungen, die wir auch bei anderen Vögeln bei ihrer Balz wiederfinden, werden von PorTielJe als ,symbolische Instinkthandlungen" oder ,Symbolhandlungen" bezeichnet.

Die Beobachtungen an der Rohrdommel (25) betrafen besonders die merkwürdigen Schutz- und Imponierstellungen, sowie die Abwehrhandlungen dieses Vogels. Wenn sich das Tier bedroht fühlt, nimmt es die, auch schon etwas bei anderen Reiheartigen vorkommende, sog. "Pfahlstellung”, an, wobei es gegen die Gefahr Front macht und die Augen darauf gerichtet hält, und sich fast unmerkbar mitdreht, wenn sich der angstauslösende Gegenstand fortbewegt. Kommt die Gefahr näher, so nimmt der Vögel eine „Imponierstellung”. ein, bei welcher Kopf und Hals mit breit ausstehenden Nackenfedern gesenkt werden und der Schnabel auf den Feind gerichtet wird. Nähert sich die Gefahr dem Vögel noch mehr, so nimmt das Tier die "Schreckstellung" ein, bei welcher es sich mit zum Vorstoss zurückgezogenem Hals und Kopf niederdruckt, aus welcher ein Angriff auf das Angesicht und die Augen des ihm Bedrohenden folgt. Ein dem Vogel vorgehaltener Stock oder die vorgestreckten Hände werden dabei von ihm ignoriert, und, wie Portielje es in Experimenten mit vorgehaltenen Pappscheiben in Form eines Kopfes zeigen konnte, greift das Tier dabei immer an, sobald es einen kopfähnlichen Umriss auf einem Torso in seiner Nähe erscheinen sieht, und richtet dabei seinen Angriff auf den Kopf. Da diese Reaktionen schon bei Nestjungen zu beobachten sind, sind sie of fenbar instinktiv festgelegt; eine Beeinflussung durch Erfahrung war dabei insoweit merkbar, als bei häufiger Wiederholung die Tiere bei dem ihnen dabei bekannten Experimentator statt mit dieser Reaktion sofort mit der Flucht antworteten, obwohl sie bei anderen Personen (Wärtern) fortfuhren, die üblichen Abwehrreaktionen zu zeigen.

Beim Kormoran (27) wurde wiederum besonders die Paarungsbiologie studiert. Hier sucht das Weibchen den Nistort aus, und beschäftigt sich mit dem Brüten und der Pflege der Jungen; das Männchen schleppt das Nestmaterial heran und ist dem Weibchen beim Brüten behilflich. Nur das Weibchen besitzt einen typischen Balzruf, welcher vom Männchen mit Balzbewegungen und dem Verständigungslaut beantwortet wird. Das Männchen holt das Nestmaterial aus dem Wasser hervor und bringt es dem Weibchen entgegen, welches dieses dann annimmt. Die Paarung wird eingeleitet durch ein gegenseitiges Anbieten von Ästchen; bei der Balz nehmen beide Tiere Stellungen ein, die von 
Portielje wieder als symbolischer Ausdruck der Haltung bei der Paarung aufgefasst werden. Bei der Paarung umfastt das Männchen liebkosend den Hals des Weibchens ${ }^{1}$ ).

Auch bei der Silbermöwe (28) wurde die Paarungsbiologie von Portielje eingehend studiert, sowohl im Garten als an den Brutplätzen in den Dünen. Hierbei wurden die verschiedenen Laute dieser Tiere beschrieben: der „Verständigungslaut”, der "Alarmschrei", der "Wutlaut", der "Zärtlichkeitsausdruck", usw. Der letztere gilt zugleich als Äusserung des Geschlechtstriebes im weiteren Sinne, beim Nestbau und als Aufforderung zur Begattung. Am imponierendsten ist bei der Silbermöwe der „Balzruf”, der aus drei Sätzen besteht, und der während der Balzzeit wiederhalt als eine Äusserung gesteigerter Vitalität ertönt. Beim Bruttrieblaut zeigt das Tier wieder eine merkwürdige, als Symbol aufzufassende Handlung: die Brust gegen den Boden stemmend, picken die Tiere eifrig vor sich hin und wackeln mit dem ganzen Körper hin und her, als ob sie eine Nestmulde machen oder Nestmaterial anordnen wollten; oder sie raffen dabei $̈$ Ästchen und Gräser und ähnliches Nestmaterial auf und werfen es wieder weg, usw. Auch später-beim Sich-niederlassen auf das Nest, beim Erblicken des herankommenden Gatten und als Zärtlichkeitsausdruck im allgemeinen tritt diese Handlung wieder auf. Eine eigentliche Balzflucht ist beim Männchen nicht zu beobachten. Weiter konnte Portielje sowohl die Paarung mit ihrem Vorspiel beschreiben, bei welcher die Initiatieve dem Männchen zukommt, während die letztere beim Nisten beim Weibchen liegt, wie auch die Lebensgeschichte der Jungen, die erst noch von den Alten ernährt werden. Und als eine merkwürdige, wenn auch nicht zur Brutbiologie gehörende, Handlung wird noch das Zerschellenlassen von Muscheln beschrieben, die der Vögel aus der Luft herunterfallen lässt, damit sie zerbrechen, wobei es unentschieden gelassen wird, ob diese Handlung einem angeborenen Instinkt oder einer erworbenen Erfahrung zugeschrieben werden muss.

Nachdem Portielje (26) noch über einige Anornalitäten des Brutinstinktes berichtet hat (ein Männchen der Paradieswachtel Lophortyx californicus setzte nach dem Tode des Weibchens die Bebrütung fort und versorgte auch die Jungen; ein von seinem Weibchen verlassenes Männchen des Löfflers Platalea leucorodia wurde brütend auf einem Stein angetroffen, und ein Männchen vom schwarzen Schwan, Cygnus atratus, zerstörte die Eier seines eigenen Weibchens, nachdem das Nest von einem Pärchen des Singschwans Cygnus cygnus besetzt gewesen war), beschrieb er noch einmal eingehend das Balzverhalten der Kampfschnepfe, Philomachus pugnax (29), und zwar wieder nach Beobachtungen die sowohl im Garten als an verschiedenen Kampfplätzen im Freien ausgeführt wurden.

1) Zusatz bei der Korrektur. Seitdem das Obenstehende geschrieben wurde, hat KorTLANDT (19a) eine Arbeit über im Anschluss an Portielje im Garten und in KormoranKolonien ausgeführter Beobachtungen über die Ausdruckbewegungen und Laute dieses Vogels publiziert. Wir können hierauf jetzt nicht näher eingehen. 
Dass die sog. Kämpfe-der Männchen der Kampfschnepfe in Wirklichkeit Balzhandlungen sind, bei welchen das Kämpfen etwas Nebensächliches ist, ist bekannt. Portiki.je konnte nun hierbei drei Phasen erkennen: eine erste, in welcher sich die Tiere trippelnd herumdrehen und mit aufgerichteten Haubenund Kragenfedern und mehr oder weniger geöffneten Flügeln vorwärts- oder rückwärtsrennen; dann die eigentliche Schaubalz, bei welcher die Tiere stillstehen und sich mit gespreiztem Schmuckgefieder und halbgeöffneten Flügeln und vorübergebeugtem Kopf niederdrucken, und drittens ein spasmatisches Zittern des Gefieders und Zucken der Flügel und des herumgebogenen Schwanzes. Die leztere Phase wird von Portielje als ein Symbol der Begattung betrachtet. Kämpfe zwischen Nachbarn am Balzplatz kommen nur in der ersten Phase vor und sind prinzipiell von der Balz zu unterscheiden. Auch dauern solche Kämpfe nur kurz. Anwesenheit der Weibchen ist für den Ausbruch eines Kampfes nicht nötig; wohl kann diese die allgemeine sexuelle Erregung steigern. Die Weibchen selbst bleiben anscheinend gegenüber diesem Verhalten der Männchen gleichgültig; die Kopulation findet meistens nicht auf dem Balzplatz, sondern in einer gewissen'Entfernung statt, wohin dem Weibchen ein paarungslustiges Männchen folgt. Hierbei kümmert sich das Weibchen nicht um die Federpracht der Männchen, und wählt nicht eins aus, sondern lässt sich oft von verschiedenen Männchen treten. Bisweilen stellt sich ein Weibchen neben einem Männchen in der 2. Phase auf, und fordert ihn durch Berührung des Gefieders zur Begattung auf. Zu einer Paarbildung kommt es jedoch nicht und die Männchen kümmern sich nicht um den Nestbau und die Pflege der Jungen. Die Männchen sind eben zu spezialisierten „Schaubalzern” geworden.

Schliesslich hat Portielje (3I) noch einen Fall von akzessorischer Promiskuität beim Männchen des Höckerschwans (C ygnnus olor), das gewöhnlich monogam ist und auch ausserhalb der Brutzeit mit seinem Weibchen in monogamer Dauerehe zusammenbleibt, beschrieben. Das betreffende Männchen duldete nicht nur ein zweites Weibchen in seinem Brutgebiet sondern kopulierte sogar mit ihr, als seine eigentliche Ehefrau mit Brüten beschäftigt war, nahe am eigentlichen Brutterritorium, sozusagen vor den Augen seines eigentlichen Weibchens, das selbst dabei gleichgültig blieb. Die Aufforderung zur Begattung ging hier vom zweiten Weibchen aus. Das Männchen kümmerte sich nicht um das Nest der zweiten Frau, wohl verteitigte er sie später selbst. Seine ersten Eier bebrütete er nicht, obwohl er wohl das eigentliche Nest überwachte und verteidigte.

Auch einige andere Untersucher haben in der Tierwelt des Gartens Material für psychologische Beobachtungen gefunden. So hat Bierens de HaAN (I) dort die Balz des Argusfasans (Argusianus grayi und Argusianus argus) studiert. Er konnte dabei drei Phasen unterscheiden: eine erste Phase, in welcher der Hahn erregt mit aufgerichteten Kopffedern umhergeht, wobei er fortwährend kleine Gegenstände vom Boden aufnimmt und wieder fallen lässt und das Weibchen verfolgt ; dann, wenn das Weibchen stehen bleibt, die Phase der Balz- 
haltung, bei welcher das Männchen plötzlich die Flügel über den Kopf hinschlägt und sie wie einen Trichter dem Weibchen vorhält, sodass dieses die vorher versteckte Flügelzeichnung zu Gesicht bekommt, und dann drittens die Phase der Tanzbewegung, bei welcher das Männchen seinen Körper bis zum Boden senkt, dabei den Schwanz hoch aufrichtet, und dies rhythmisch etwa ro mal wiederholt. Das Weibchen bleibt diesem Allen gegenüber wieder anscheinend gleichgültig und reagiert nur dadurch, dass es stehen bleibt. Dieses Stehenbleiben des Weibchens scheint für die Ausführung der Balz notwendig zu sein : ein Männchen eines Argusianus argus, das nicht mit einem Weibchen seiner eigenen Art, sondern mit einem Lophura-weibchen zusammen war, welches nicht stehenbleiben wollte, balzte daher vor seinem Futternapf oder Trinkgefäss, einmal sogar vor einem Kohlblatt, und versuchte danach mit diesen Gegenständen zu kopulieren.

Fräulein Holzapfel (I8) hat im Garten der Gesellschaft besonders die Bewegungsstereotypien, d. h. die automatenhaften mehr oder weniger zwangsartig ausgeführten Bewegungen studiert, die bei im Garten gehaltenen Säugern beobachtet werden können und die von einigen Autoren als aus Spieltrieb hervorgegangene lustbetonte Bewegungen, von anderen dagegen als ein Ausdruck psychischer Störungen betrachtet wurden. Besonders richtete sie ihre Aufmerksamkeit auf zwei solcher Bewegungen: das Hin- und Herlaufen in geraden Bahnen an einer Käfigwand entlang, und das Laufen in einer 8-förmigen Bahn, in sog. "Achterschlingen". Sie fand, dass diese Bewegungen bedingt und gefördert werden durch affektive Erregungen, die teilweise von den Menschen hervorgerufen werden und dann einen Zusammenhang mit dem Grad der Eingewöhnung und der Zahmheit des Tieres erkennen lassen. Das Hin- und Herlaufen, stets mit Wendung nach der Gitterwand hin, wurde besonders bei zwei Füchsen beobachtet und von der Autorin bei diesen scheuen Tieren als eine "Flucht an Ort", bei anderen zahmeren Tieren (Hyänen, Dingo), als ein sozial bedingtes Streben (zu den Artgenossen hin) oder ein Streben zu dem futterspendenden Menschen hin, gedeutet. Aus diesem Hin- und Herlaufen entwickelt sich das Laufen in Achterschlingen, wie sehr überzeugend an Hand von Skizzen ihrer Bahnen bei Dingos und einer Hyäne gezeigt werden konnte. Verschiedene Faktoren (Fluchttendenz, Neugier, soziale Triebe) wirken dazu zusammen, wobei eine steigende Erregung eine flachere Bahn, Beruhigung dagegen eine Verbreiterung und schliesslich Auflösung der 8-Form zur Folge haben. Bei einem Schakal waren die stereotype Bahn und ihre Form durch die Anwesenheit von Menschen bedingt, und es zeigte sich hier eine interessante gleichzeitige Wirkung von entgegengesetzten Strebungen: Flucht einerseits und Neugier andererseits, die zusammen die Form der Bahn bestimmten.

Schliesslich hat Fräulein Heubel (17) noch Beobachtungen und einfache Versuche an einem Känguruh (Macropus giganteus) angestellt. Durch besondere Behandlung während einer Krankheit war das Tier so zahm geworden, dass es sogar seinem Wärter und der Experimentatorin mit dem Mund 
Pindanüsse aus ihrem Munde nahm. Wurde dies aber im Aussenkäfig statt im Innenkäfig des Verschlages versucht, so floh das Tier. Die zutrauliche Reaktion dem Wärter gegenüber war also an einen besonderen Ort gebunden. Bei einer Untersuchung über die Bedeutung der verschiedenen Sinne für die Futtererwerbung zeigte es sich, dass der Geruchssinn für das genaue Erkennen des Futters und die Unterscheidung zwischen Futter und Scheinfutter der wichtigste Sinn ist, obwohl das Tier auch schnell lernen kann, sich nach optischen Merkmalen des Futters zu richten, sogar, wenn die Unterschiede zwischen bevorzugtem und weiniger bevorzugtem Futter sehr gering gemacht waren. Auch strebt das Tier nach dem bevorzugten Futiter und sucht nach diesem, wenn es weiniger bevorzugtes Futter gefunden hat; und gelernt hat, dass das bevorzugte Futter zu finden, ist. Versuche über Farbendressur (Weiss gegen Schwarz) gelangen in kurzer Zeit; Versuche über das Suchen nach vor seinen Augen verstecktem Futter (siehe die unten zu beschreibenden Versuche Bierens de HaANs) gelangen nur, wenn das Futter mittels des Geruchssinnes bemerkbar war. In Versuchen, in welchen das Tier, um Futter zu bekommen, ein Gewicht mit den Vorderpfoten hinaufziehen musste, zeigte es sich, dass das Tier imstande war, in dieser Weise ein Gewicht bis zu $3 \mathrm{~kg}$ mit den Vorderpfoten zu heben.

\section{IM AQUARIUM DER GESELLSCHAFT ANGESTELLTE BEOBACHTUNGEN}

Das an Tierleben so reiche Aquarium der Gesellschaft ist bis jetzt noch viel zu wenig für tierpsychologische Beobachtungen und Experimente ausgenutzt worden. Nur Portielje (30) hat dort einige Beobachtungen über eine besondere Weise des Futtersuchens des kleinen Brackwasser-Anemone Diadumena cincta gemacht. Wenn diese Tiere hungrig sind, führen sie spontanen Suchbewegungen aus, indem sie ihre sehr lang ausgestreckten und verdickten Tentakeln, deren Fnde in Form eines Nadelkopfes angeschwollen ist, durch das umgebende Wasser herumbewegen. Auch bei einigen anderen Anemonen liessen sich diese of fenbar zum Futtererwerb dienende Handlungen beobachten.

\section{IM TIERPSYCHOLOGISCHEN LABORATORIUM DER GESELLSCHAFT ANGESTELLTE BEOBACHTUNGEN}

Auch das eigentlich mehr für Experimente eingerichtete Laboratorium bot Gelegenheit zu einigen Beobachtungen über die Instinktäusserungen und Gewohnheiten der Tiere.

So studierte Bierens De HAaN (7) dort das sog. "Waschen” der Waschbären (Procyon lotor). Es ergab sich dabei, dass dies nicht, wie wohl gemeint wurde, mit Reinlichkeit oder Feuchtigkeitsbedürfnis zu tun hat, sondern eine Art Spiel ist, das sowohl mit essbaren als nicht-essbaren Gegenständen ausgeführt wird, und einerseits zu einem Einweichen des Gegenstandes im Wasser 
vereinfacht, andererseits auch ausserhalb des Wassers ausgeführt werden und in Rollen, Kratzen oder Beissen des Gegenstandes übergehen kann. Besonders glatte und kalte Gegenstände sind als Waschobjekt beliebt, während dagegen Gegenstände mit einer rauhen Oberfläche mehr gekratzt werden. Die Neigung zum Waschen oder Kratzen ist bei den Waschbären individuell sehr verschieden. Das Waschen und die verwandten Tätigkeiten sind also als „sensorische Spiele". zu betrachten, d. h. als Spiele, die bezwecken, lustvolle Empfindungen zu erwecken, und die wir in anderer Form auch bei anderen Tieren (Affen, Hunden, usw.) beobachten können.

In Anschluss an ihre oben beschriebenen Wahrnehmungen über Bewegungsstereotypien im Garten hat Fräulein Holzapfel (19) im Laboratorium noch Beobachtungen über solche. Bewegungsgewohnheiten bei einem Gürteltier (Dasypus villosus) angestellt. Das Tier hatte die Gewohnheit, in den Nachmittagsstunden im Käfig schnell hin- und herzulaufen, hauptsächlich an der linken Wand entlang, welche Bewegung es nur von Zeit zu Zeit durch Schnüffeln oder scharrend-kratzende Bewegungen unterbrach. Es wurde vermutet, dass diese Bewegungen Ausdruck einer Erregung waren, die durch das Fehlen der für das biologisch zu Wühlen bestimmte Tier benötigte Erde verursacht wurde. Nachdem es sich gezeigt hatte, dass in den Käfig gebrachte Gegenstände das Tier nur vorübergehend von seiner Laufgewohnheit ablenkten, bezw. die Bahn des Tieres beeinflussten, und dass auch Käfigwechsel zwar den Weg des Tieres änderte, aber das Herumlaufen in keiner Weise verminderte, konnte die erste Vermutung durch Hineinbringen des Tieres in einen Käfig mit einer $20 \mathrm{~cm}$ höhen Sandschicht bewiesen werden: die Laufgewohnheit hörte auf, und das Tier grub sich jede Nacht ein Loch in einer Ecke des Käfigs und schlief dort zusammengerollt. Als dann allmählich der Sand wieder weggenommen wurde, fing das Tier am Ende an, auch in diesem Käfig umherzutraben, und als es dann in seinen ursprünglichen Käfig zurückgebracht wurde, lief es dort wieder wie vorher an der linken Wand entlang. Die Laufgewohnheit war also Ausdruck einer Dauererregung; die durch des Fehlen eines biologisch seinen Bedürfnissen angepassten Bodens hervorgerufen wurde.

\section{IM TIERPSYCHOILOGISCHEN LABORATORIUM DER GESELL- SCHAFT AUSGEFÜHRTE EXPERIMENTE}

\section{a. Experimente über die Wahrnehmungen der Tiere}

Die im Laboratorium ausgeführten Experimente lassen sich in vier Gruppen einteilen, je nachdem es sich dabei um die Besonderheiten der tierischen Wahrnehmungen, oder um ihr I,ernvermögen und ihre Intelligenz, um das Erilernen bestimmter Handlungsgewohnheiten oder um den Einfluss ihres Charakters auf ihre Handlungen und ihr Lernvermögen handelt. Wir fangen hier mit der ersten Gruppe an.

Bierens de HaAN und Frima (5) untersuchten den Farbensinn der 
Lemuren. Dies war darum wichtig, weil es bekannt war, dass niedere Affen einen Farbensinn haben, der ganz mit dem menschlichen übereinstimmt, während die meisten anderen Säuger entweder farbenblind sind oder eine so schwache Farbenunterscheidung besitzen, dass sie doch praktisch als farbenblind gelten dürfen. Das Ergebnis war, dass ein Lemur mongoz jede der vier Hauptfarben (Rot, Grün, Gelb, Blau) mit je einer Gruppe von Graupapieren bestimmter Helligkeit verwechselte, während er andererseits die Dressur von Rot gegen Blau sehr leicht auf eine von Grün gegen Gelb übertrug. Dieses Tier sah die Farben also als Graunuancen und war also farbenblind. Bei einem zweiten Tier derselben Art konnte nur die Unterscheidung von Blau untersucht werden. Diese Farbe wurde zwar wieder erst mit bestimmten Graunuancen verwechselt, aber wurde doch bei Fortsetzung der Versuche von diesem Tiere allmählich besser unterschieden, sodass der Schluss sein muss, dass dieses Tier doch über ein, wenn auch sehr schwaches, Farbensehen verfügte.

Bierens de HaAN (2) hat sich dann weiter, in Anschluss an vorher an anderer Stelle von ihm ausgeführte Untersuchungen, die Frage gestellt, ob und inwieweit Affen zwei Gegenstände von ungefähr gleicher Grösse aber verschiedener Form leichter unterschieden als zwei Gegenstände von gleicher Form, aber ungleicher Grösse. Dazu wurden zwei Schweinsaffen (Nemestrinus nemestrinus) erst auf den Unterschied zwischen zwei gleichförmigen Kegeln mit einer Höhe von I 6 bezw. I I cm, danach auf den kleineren Kegel gegen einen Würfel mit $7 \mathrm{~cm}$ Seitenlänge dressiert. Bei der ersten Dressur war nach 125 Versuchsserien (1250 Einzelversuchen) bei keinem vom beiden Tieren Lernerfolg zu beobachten; dagegen gelang die Dressur auf Kegel gegen Würfè sehr schnell (in 270 bezw. 350 Versuchen). Als dann die ersten Versuche mit Kegeln von 16 und $8 \mathrm{~cm}$ Höhe wiederholt wurden, zeigte es sich, dass der grössere Kegel eine grössere Anziehungskraft auf beide Tiere ausübte als der kleinere, wahrscheinlich, weil sie die Eigenschaften des Dressurobjektes auf die Belohnung übertrugen. Schliesslich lernte das Tier, das auf den grösseren Kegel dressiert wurde, dies in 350 Versuchen, und dasjenige, das auf den kleineren dressiert war, in 600 Versuchen. Als diese Dressur einmal gelungen war, gab es keine besondere Schwierigkeiten mehr, die Tiere dann auf den kleineren Unterschied zwischen dem Dressurkegel und dem Kegel van I I cm zu dressieren. Immerhin zeigte es sich, dass die Dressur auf zwei Gegenstände ungleicher Form bei Affen auffallend viel leichter ist als die auf zwei Gegenstände von ungleicher Grösse.

Bei derselben Gelegenheit hat Bierens de HaAN (2) auch untersucht, ob Affen zweidimensionale Abbildungen von ihnen bekannten Dressurobjekten wiedererkennen. Dazu wurden ein Schweinsaffe und ein Mangabe (Cercocebus fuliginosus) entweder auf den mittleren Kegel oder den Würfel der vorherigen Versuche in der Kombination: Kegel-Würfel, dressiert. Der Schweinsaffe lernte in $35^{\circ}$ Versuchen nach dem Würfel hinzugehen; für den Mangaben waren 700 Versuche erforderlich, die andere Dressur ziemlich gut zu vollenden. Als 
dann die Gegenstände durch Zeichnungen von derselben Grösse ersetzt wurden, war der Schweinsaffe vollkommen verwirrt und machte ungefähr $50 \%$ Fehler, wodurch er bewies, dass er das Dressurobjekt in den Zeichnungen nicht wiedererkannte. Der weniger gut beobachtende Mangabe dagegen machte nicht mehr Fehler als zuvor. Fraglich ist, inwieweit man hier von einem Wiedererkennen sprechen darf, und das Tier sich nicht auf ein bestimmtes gemeinschaftliches Element in den beiden Wahrnehmungen (z.B. das der "Spitzheit" des drei dimensionalen Gegenstandes und seiner zweidimensionalen Abbildung) dressiert hatte. Ein Versuch, das letztere Tier noch auf den Würfel umzudressieren, misslang aus unbekannten Ursachen in 1650 Versuchen.

Verschiedene Autoren hatten sich gef ragt, welches eigentlich das Merkmal ist, nach welchem ein Tier sich richtet, das wir auf eines von zwei, nur in quantitativer Beziehung verschiedenen Objekten (z.B. zwei Graunuancen von verschiedener Helligkeit) zu dressieren versuchen. Lernt das Tier dann ein Grau von einer bestimmten absoluten Helligkeit wählen, oder wählt es das dunklere bezw. hellere der beiden Graunuancen, also relativ? Man kann dies ermitteln, indem man nach Vollendung der Iressur dem Tier zwei andere Objekte darbietet, von welchen das eine einem der beiden Dressurobjekte, (im Falle der Graunuancen also das dunklere oder hellere Grau) ähnlich ist, das andere dagegen ebenso viel dunkler bezw. heller ist als bei der ursprünglichen Dressur das eine dunkler bezw. heller war als sein Gegenobjekt. Bierens de HAAN hat dies nun (3) bei zwei Schweinsaffen untersucht. Bei Versuchen, bei welchen das eine Tier auf das hellere, das andere auf das dunklere von zwei Graupapieren der Heringschen Serie dressiert wurde, wählte in den Kontrollversuchen bei Verschiebung nach der positiven Seite das eine Tier in $90 \%$ und das andere in $60 \%$ der Versuche absolut, bei Verschiebung nach der negativen Seite das eine in $85 \%$ und das andere in $82,5 \%$ relativ. Die Versuche wurden dann wiederholt, wobei als Dressurobjekt zwei kleine schwarze Kreise, in verschiedener Entfernung auf ein weisses Papier geklebt, dargeboten wurden. Das erste Tier wurde nun auf die grössere, das andere auf die kleinere Entfernung der Kreise dressiert. Bei den Kontrollversuchen wählten nun beide Tiere bei Verschiebung nach der positiven Seite etwa in $50 \%$ der Versuche absolut und in $50 \%$ relativ; bei Verschiebung nach der negativen Seite bezw. in $72,5 \%$ und in $65 \%$ relativ. Schliesslich wurde das erste Tier noch auf das grössere von zwei schwarzen Dreiecken dressiert; in den Kontrollversuchen wählte es dann bei Verschiebung nach der positiven Seite in $50 \%$ absolut und in $50 \%$ relativ und bei Verschiebung nach der negativen Seite in 67,5\% relativ. Für die Deutung dieser Tatsachen sei nach der Originalarbeit selbst verwiesen; gegenwärtig wird diese Frage nach der Wahl nach absoluten oder relativen Merkmalen aufs neue im Laboratorium untersucht, welche Versuche zur Zeit noch nicht abgeschlossen sind.

Schliesslich hat Sмıтн (32) noch untersucht, welches die Bedeutung von Form und von Farbe in der Wahrnehmung von Affen ist. Dazu. wurden sechs 
junge Schweinsaffen von etwa gleichem Alter in einem Wahlapparat darauf dressiert, bei Darbietung eines roten Dreiecks und eines blauen Kreises, beide auf weissem Grunde, das Dreieck bezw. den Kreis zu wählen. Als diese Dressur vollendet war, wurde zur Analyse des Dressurprozesses dieser Form-FarbeKomplex geändert und gespalten, indem entweder in einer der Figuren, oder in beiden, Änderungen in der Form oder der Farbe angebracht wurden. So wurden z.B. ein ganz rotes und ein ganz blaues Papier oder ähnlich gefärbte Rechtecke oder Ovale auf. weissem Grund dargeboten, oder es wurden ein Dreieck und ein Kreis dargeboten, deren Umrisse nur gezeichnet waren oder die beide eine neue (grüne) Farbe hatten. Auch wurden Form und Farbe gewechselt, also ein blaues Dreieck gegen einen roten Kreis dargeboten, oder es wurde der ganze Dressurkomplex geändert, und die Dressurfigur gegen eine andere von derselben Form oder Fărbe dargeboten; auch wurde die positive oder die negative Figur ganz weggelassen und durch eine weisse Karte ersetzt. Und bei denjenigen Tieren, für welche das Dreieck die positive Figur gewesen war, wurde die Lage und die Form des Dreiecks noch geändert, dieses z.B. umgekehrt dargeboten, usw. Die Folgen aller dieser Änderungen wurden in Zahlen und Kurven ausgedrückt, die sich schwierig kurz zusammenfassen lassen. Als allgemeines Resultat kann erwähnt werden, dass die Bedeutung von Form bezw. Farbe individuell sehr verschieden ist: einige Tiere waren hauptsächlich auf Form, andere auf Farbe dressiert, während bei wieder anderen Form und Farbe etwa gleiche Bedeutung hatten. Auch die Bedeutung der positiven und der negativen Karte war sehr verschieden : einige Tiere hatten gelernt, die positive Figur zu suchen, andere, der negativen zu entweichen. Man darf also die bei einem bestimmten Tier erworbenen Ergebnisse nicht generalisieren, und es zéigte sich, dass ein solcher Dressurprozess sich viel verwickelter gestaltet als wohl angenommen wird.

\section{b. Experimente über das Lernvermögen und die Intelligenz der Tiere}

Kuiper (20) hat bei weissen Ratten untersucht, ob das Erlernen eines Labyrinthes schneller verläuft, wenn man die Tiere täglich übt, oder wenn man sie nur zweimal wöchentlich das Labyrinth durchlaufen lässt. Als Lernapparat wurde dazu ein sog. quadratisches Labyrinth von CARR gewählt ; beide Gruppen Ratten umfassten 4 'Tiere. Ergebnis war, dass die Ratten, die nur zweimal wöchentlich geübt wurden, das Labyrinth schneller erlernten als die täglich geübten Tiere; die erste Gruppe benötigte durchschnittlich I6 bis I7 Versuche, die zweite 27 bis 28 Versuche, um das Labyrinth zu erlernen. Ähnliches war aưch schon bei anderen Tieren und bei Kindern gefunden worden.

In einer Reihe Versuche hat Bierens DE HAAN dann das konkrete Verständnis einer Anzahl Versuchstiere unter verschiedenen Umständen geprüft.

Vierzehn Versuchstieren (6 zu 4 Arten gehörenden Affen, 3 Lemuren, 2 Waschbären, 2 Nasenbären und ein Eichhörnchen, also I4 Tiere, die zu dem- 
selben biologischen Typus, nämlich dem der höheren Baumtiere mit als Hände benutzbaren Vorderextremitäten, gehörten) wurden acht einfache Aufgaben gestellt, bei welchen sie durch eine einfache, auf Verständnis beruhende Handlung Futter erwerben konnten, das von dem Experimentator in verschiedenen Weisen vor ihren Augen versteckt wurde $(4,8)$. So mussten sie zu diesem Zwecke einen Blumentopf umkehren, unter welchem Futter niedergelegt war, oder die Hand des Experimentators öffnen, in welcher er Futter verborgen hielt, Futter aus seiner Tasche hervorholen, eine Büchse öffnen oder einen Deckel aufheben, Futter an einem Bindfaden in einer Büchse heranziehen oder von einem. Brett herunterziehen, usw. Die 'Tiere verhielten sich, wie wohl zu erwarten war, diesen Aufgaben gegenüber sehr verschieden. Während in einigen Fällen sofort Verständnis der auszuführenden Handlung vorhanden war, und die 'Tiere die Aufgabe also primär lösten, bildete sich dieses Verständnis in anderen Fällen nur allmählich sekundär aus, und fehlte es in wieder anderen Fällen völlig und dauernd. Nach den Ergebnissen liessen sich die untersuchten 'Tiere in drei Gruppen ordnen : eine, bei welcher immer oder fast immer einer primäre Lösung erfolgte, eine, bei welcher die Lösung der Aufgabe oft erst sekundär geschah oder ganz ausblieb, und eine dritte, bei welcher oft garnicht mehr nach dem aus ihren Augen verschwundenen Futter gesucht wurde. Merkwürdig war es hierbei, dass die Zusammenstellung der drei Gruppen nicht immer mit dem übereinstimmte, was man nach der systematischen Einteilung erwarten durfte, und z.B. die L,emuren höher standen als einige der Affen, und die benutzten Raubtiere wieder höher als diese Affen und die Lemuren.

Diese Inkongruenz zwischen ihrer Stelle im System und ihrer Intelligenz trat noch mehr hervor, wenn Bierens de HAAN (15) das Verständnis dieser Tiere mit dem verglich, was von anderen (KöHLER) früher bei Schimpansen gefunden war. Zwei Aufgaben, die von KöhLERs Schimpansen nur mit Mühe gelöst wurden, nämlich das Wegschieben einer sie hindernden Kiste, und das Weitergeben eines an einen Gegenstand befestigten Bindfadens von der einen Hand in die andere durch die Maschen des Käfigs hindurch, welcher Bindfaden so gedreht werden sollte, damit die in der Mitte des Bindfadens angebundene Frucht in den Bereich des Tieres kam, wurden von den meisten der obengenannten Tiere schneller und besser gelöst als. von den Schimpansen. Dies zeigt noch einmal, dass der Grad der Intelligenz nicht einfach mit der morphologischen Entwicklung des Tieres und seiner Stelle im System zusammenhängt.

Bei einem der Versuchstiere, einem Weisschulterkapuzineraffen (Cebus hypoleucus), wurde weiter geprüft, ob er imstande war, zu verstehen, dass er durch Heranschleppen einer Kiste, bezw. durch Aufeinanderstapeln mehrerer solcher Bauelemente unter eine hoch aufgehängte Frucht, die Frucht selbst erreichen könne (6). Die Erwartung des Experimentators wurde dabei nicht getäuscht : allmählich, bei gradueller Erschwerung der Aufgabe (Höherhängen der Frucht) zeigte das Tier Verständnis: erst, eine grosse Kiste bis unter die 
Frucht zu schleppen oder zu kanten; dann, eine zweite Kiste auf eine schon an richtiger Stelle stehende zu stellen; dann, zwei freistehende Kisten heranzuziehen und zu stapeln; und schliesslich, mit drei Kisten oder ähnlichen Elementen $z u$ bauen, erst wieder, wenn eine oder zwei derselben bereits unter der Frucht standen, und schliesslich, wenn alle drei Kisten an verschiedenen Stellen im Käfig zerstreut standen. Auch nicht direkt wahrnehmbare (in seinem Schlafraum versteckte) Gegenstände wurden gesucht und dann zum Bau herangezogen. Das Tier verstand es auch, den Stock als Kletter-, Schlag- oder Wurfwerkzeug zu benutzen und kombinierte dies bisweilen mit dem Heranschleppen einer Kiste. Es konnte in diesen Leistungen mit den besten bis jetzt bekannten Schimpansen auf eine Reihe gestellt werden und in seinen Leistungen kam ihm bis jetzt noch kein anderer niederer Affe gleich.

Später (II) wurden ähnliche Bauversuche noch mit einigen Wasch- und Nasenbären angestellt. Ergebnisse wurden dabei nur bei einem ausgewachsenen Nasenbären erzielt. Dieses Tier lernte sehr allmählich, teilweise mit Hilfe des Versuchsleiters, von jeder Stelle des Käfigs aus eine Kiste unter ein hochgehängtes Stück Brot heranschleppen, um sie zu besteigen. Wenn diese Leistung auch derjenigen des vorherigen 'Tieres sehr nachstand, war sie doch bemerkenswert als der erste Fall eines solchen Heranschleppens einer Kiste bei einem Nicht-Primaten.

Während es sich bei diesen letzten Versuchen um ein Verständnis der Folgen eigener Wirkungen bei den 'Tieren handelte, kann die Intelligenz der Tiere sich auch darin äussern, dass sie imstande sind, bestimmte räumliche Beziehungen zu erfassen. Yerkes hatte diese Fähigkeit zù messen versucht, indem er verschiedene 'Tiere (Affen, Krähen, Schweine, Ratten) darauf zu dressieren versuchte, aus einer Reihe von gleichartigen Elementen dasjenige auszuwählen, das sich durch seine bestimmte Lage in der Reihe (erstes von links, zweites von rechts, usw.) auszeichnete ${ }^{1}$ ). Hierbei hatten aber die Affen ihn in ihren Leistungen, mit denen der anderen Tiere verglichen, enttäuscht. BIERENS DE HAAN (14) stellte sich nun die Frage, ob die Ursache dieser relativ schlechten Ergebnisse bei Affen vielleicht die war, dass Yerkes eine für Affen wenig glückliche Methode gewählt hatte (die Affen sollten, wie die anderen Versuchstiere, in ein bestimmtes von einer Reihe ähnlicher, auf dem Boden aufgestellter Kästchen hineingehen, und bekamen nur Futter, wenn sie das richtige gewählt hatten, während sie in anderen Fällen einige Zeit eingesperrt blieben). Es wurde für möglich gehalten, dass bei Affen bessere Ergebnisse erzielt werden konnten, wenn sie bei dieser Aufgabe nach hangenden Kistchen greifen sollten, und vielleicht noch mehr nach übereinander statt nach nebeneinander gehängten Kistchen. Beide Annahmen erwiesen sich jedoch als unberechtigt: Bei drei Versuchstieren (einem Mangabe, einem Java-affen und einem Rhesus-

1) R. M. YERKEs. Methods of exhibiting reactive tendencies characteristic of ontogenetic and phylogenetic stages. Journ. of animal Behavior, VII, 1917. 
Affen) waren mit dieser Methode die Ergebnisse bei zwei verschiedenen Aufgaben nicht besser als die, welche YERKEs bei ähnlichen Tieren erhalten hatte. Warum es den Affen so relativ schwer fällt, räumliche Beziehungen dieser Art zu erfassen, ist nicht bekannt.

Schliesslich hat Bierens De HAAN (9) noch das sog. "Schöpfen" des Stieglitzes studiert. Diese Tiere können bekanntlich erlernen, ein hängendes Eimerchen mit Wasser oder ein auf einem geneigten Brettchen laufendes Wägelchen mit Futter heraufzuziehen, wobei sie dann genötigt sind, das mit dem Schnabel an einem Bindfaden teilweise herangezogene Wägelchen durch Drücken des Fusses auf den Bindfaden einen Moment festzuhalten, um dann mit dem Schnabel den Bindfaden an einer weiteren Stelle zu ergreifen. Bierens DE HAAN fand nun bei drei Stieglitzen, dass es sich hierbei für den Vögel um zwei gesonderte Aufgaben handelt; erstens zu verstehen, dass durch Ziehen am Bindfaden das Wägelchen mit dem Futter näher kommt, und zweitens zu verstehen, dass er mit dem Fuss den Bindfaden festdrücken muss, um zu verhindern, dass das Wägelchen zurückrollt. Die Begabung dafür ist bei den Tieren individuell sehr verschieden: während ein Tier fast direkt verstand, das Wägelchen am Bindfaden heranzuziehen und den Fuss auf den Bindfaden zu setzen, konnte ein zweites Tier sogar das Heranziehen des Wägelchens nicht erlernen, während ein drittes Tier zwar das Wägelchen sofort heranzog, aber erst sekundär erlernte, den Fuss auf den Bindfaden zu drücken. Obwohl auch angeblich einige andere Singvögel imstande sind, dieses Kunststück zu lernen, hatten mit zwei Grünlingen, zwei Buchfinken, zwei Hänflingen und zwei Zeisigen angestellte Versuche nur einen geringen Erfolg, und konnten diese Tiere höchstens lernen, das Wägelchen heranzuziehen, ohne dass es bei ihnen zu einem Festhalten mit dem Fuss kam.

\section{c. Experimente über das Erlernen bestimmter Handlungsgewohnheiten}

Von einigen ausländischen Forschern war behauptet worden, dass Affen und Hühner imstande seien, Zahlbegriffe zu bilden und damit einfache Rechenleistungen auszuführen. Bierens De HAAN (12) hat die Versuche, die Anlass zu diesen Behauptungen gaben, kritisiert, und dann selbst an Versuchen mit einem Java-Affen gezeigt, dass das, was die betreffenden Autoren erhielten, nichts Anderes als eine Dressur auf bestimmte Rhythmen der Handlung war. Sein Versuchstier lernte durch Darbietung einer Büchse mit bezw. ohne Futter zweimal diese Büchse umkehren und sie das dritte Mal nicht zu berühren. Aus Kontrollversuchen mit Darbietung der Belohnung in anderer Menge (zwei Stückchen, bezw. gar nichts) zeigte es sich dann, dass die Reaktionen des Tieres in diesen Fällen nicht auf Zählen beruhten, sondern nicht-erfolgbringende Handlungen (das Umkehren der Büchse, wenn kein Futter unter diese gelegt war) nicht in den Rhythmus einbezogen wurden; auch zeigte es sich, dass bei Verlängerung des Intervalles zwischen zwei Darbietungen von to auf 20 und 
30 Sekunden der Rhythmus allmählich verloren geht. Das Tier lernte danach sehr leicht, nach einem einfacheren Rhythmus (einfacher Alternation) zu handeln, und diesen einfacheren Alternationsrhythmus besser als den verwickelteren, wenn die Intervalle zwischen den Einzelhandlungen verlängert wurden.

Später haben Bierens de HaAN und Kooyman (13) noch untersucht, wie solche Handlungsrhythmen beim Affen gebildet wurden. Das Versuchstier war diesmal ein Schweinsaffe (Nemestrinus nemestrinus), der den verwickelteren "+ + + - -Rhythmus erlernen sollte, also erst dreimal positiv reagieren und dann einmal negativ. Die Dressur verlief in drei Etappen. Erst reagierte das Tier bei allen Darbietungen nur positiv; dann fing es an, auch negativ zu reagieren, wonach die negativen Reaktionen erst bei der zweiten und dann bei der dritten Darbietung verschwanden. Am längsten blieb als Fehler das unrichtige positieve Reagieren bei der 4. Darbietung übrig. Als das Tier also den ,,jaja-ja-nein"-Rhythmus erlernt hatte, wurde die Belohnung wiederum in anderen Mengen dargeboten, und schliesslich wurde das Tier auf einfachere Rhythmen umdressiert. Beim ersteren zeigten sich zwei Tendenzen: Folgen des andressierten Handlungsrhythmus und Nichtmiteinbeziehen der nicht-belohnten Handlungen in diesen Rythmus. Die Dressur auf einen einfacheren Rhythmus, nachdem ein verwickelterer gelernt war, verlief im allgemeinen sehr leicht.

\section{d. Experimente über den Einfluss ihres Charakters auf das Lernvermögen und das Handeln der Tiere}

Dass der Charakter der Versuchstiere einen Finfluss auf ihr Lernvermögen hat, zeigte sich deutlich in einer von Bierens de HaAN und MEyKNecht (IO) angestellten Untersuchung. Für eine Dressur auf das dunklere von zwei Graupapieren, die bei Schweinsaffen ohne Schwierigkeit gelungen war (siehe oben S. 43), wurde einmal ein Mangabe (Cercocebus fuliginosus) gewählt, welcher dieser Dressur besondere Schwierigkeiten entgegensetzte. Der Grund hiervon war, dass dieses Tier zu einem Tiertypus gehörte, welcher von SzyMANSKI ${ }^{1}$ ) als der „motorische” im Gegensatz zu dem „sensoriellen” Typus bezeichnet war, einem Tiertypus, dessen Aktivität sich hauptsächlich auf motorischem Gebiete äussert, das sofort handelt, ohne erst zuzusehen und dadurch mehr Neigung zeigt, sich von bestimmten Bewegungsgewohnheiten leiten zu lasen (z.B. Wahl der linken oder rechten Karte), als dass es dazu kommt, seine Wahl nach den optischen Merkmalen der Dressurobjekte zu treffen. Das benutzte Tier schwankte nun fortwährend hin und her zwischen Rechtswahl und Linkswahl, sodass nach 14 Versuchstagen (920) Versuchen, es noch immer ungefähr $50 \%$ Fehler machte. Um es dann dazu zu bringen, seine motorisch bedingte Wahl in eine sensorielle zu verwandeln, wurden die optischen Merkmale der Dressurobjekte verstärkt und die Graupapiere durch ein weisses

I) J. S. Szymanski. Motorische und sensorielle Tiertypen. Biol. Zentr., XL, 1920. 
gegen ein schwarzes Papier ersetat. Dies hatte aber keinen Ërfolg: in 470 Versuchen in 6 Tagen blieb sein Fehlerprozentsatz nur wenig unter $50 \%$. Dann wurde der Unterschied zwischen den Dressurobjekten noch mehr verstärkt, indem die grauen Papiere durch zwei farbige ersetzt wurden (Rot gegen Gelb). Erst jetzt war allmählich Lernen bemerkbar, obwohl es noch I7Io Versuche in I8 Versuchstagen kostete, ehe das Tier fehlerlos gelernt hatte, das rote Papier zu wählen. Dann wurde der umgekehrte Weg eingeschlagen, und das Tier erst wieder auf Schwarz gegen Weiss, dann auf das Dunklere von den zwei Graupapieren dressiert. Die Unterscheidung Schwarz-Weiss wurde nun ziemlich schnell erlernt ( 800 Versuche in 8 Tagen), die ursprüngliche Dressur dann auch, wenn auch langsamer (I700 Versuche in 18 Tagen). So war denn längs einem langen Umweg schliesslich doch das erreicht, was bei einem mehr sensoriellen Schweinsaffen in nur 240 Versuchen gelungen war; wohl ein Beweis dafür, wie stark der Charakter des Versuchstieres sich einer bestimmten Dressur widersetzen oder diese unterstützen kann.

Der Einfluss des Charakters der Tiere auf ihre Handlungen zeigte sich schliesslich noch in Untersuchungen, die Bierens de HaAN und Heubel (i6) über die Futtervorliebe von Affen und die Beeinflussung derselben durch verschiedene Eingriffe anstellten. Bei sechs, zu drei verschiedenen Affentypen gehören Versuchstieren (zwei Makaben, zwei Meerkatzen und zwei Kapuzineraffen) wurde erst die Rangfolge der Vorliebe für 7 bezw. 5 verschiedene Futterarten prozentual bestimmt. Hierbei zeigte es sich, dass die Charaktere der Tiere sehr verschieden waren, und zwischen zwei Polenpaaren: Gefrässigkeit und Wählerischkeit eınerseits, und motorischer und sensorieller Veranlagung andererseits schwankten. Hiernach wurden dann die Bedingungen, unter welchen die Tiere zu wählen hatten, in verschiedener Weise geändert: das weniger bevorzugte Futter wurde in doppelter Menge dargeboten, oder das mehr bevorzugte in weiterer Entfernung niedergelegt, oder mit einem kleinen Glas bedeckt, sodass die T'iere sich etwas mehr Mühe geben mussten, um es zu bekommen; oder es wurde durch Bedeckung mit einem schwarzen Glas unsichtbar gemacht. Auch wurden beide Futterarten mit einem schwarzen Glas bedeckt und geprüft, ob die Tiere, wenn sie bei Umkehrung eines Glases das weniger geliebte Futter fanden, nach dem mehr geliebten weiter suchten. Alle Änderungen in der Wahl, die durch diese Änderungen in den Bedingungen hervorgerufen wurden, wurden in Zahlen ausgedrückt. Es erwies sich nun als möglich, diese Zahlen im allgemeinen mit den oben erwähnten Charakterunterschieden in Zusammenhang zu bringen. Je wählerischer die Tiere waren, um so grösser waren die relativen Vorliebezahlen; je grösser die Gefrässigkeit war, desto mehr wurde die Wahl durch Darbietung einer doppelten Menge des weniger bevorzugten Futters geändert; je mehr das Tier sensoriell veranlagt war, um so weniger machte es sich daraus, sich etwas mehr Mühe geben zu müssen und desto besser suchte es im allgemeinen noch nach verschwundenem Futter, usw. Wenn auch nicht in allen Fällen der Zusam- 
menhang zwischen Charakter und Handlung ganz eindeutig zutage trat (vielleicht, weil ein noch nicht entdeckter Charakterzug das Handeln mit beeinflusste), so war doch der Einfluss des Charakters in diesen Wahlen unverkennbar.

Es waren also an vielen verschiedenen Versuchstieren angestellte Beobachtungen und Versuche mit vielerlei Fragestellungen, die in dem Garten der jubilierenden Gesellschaft in den letzten 20 Jahren statthatten. Es gereicht der Leitung der Kön. Zool. Gesellschaft zur Ehre, dass sie ihr Tiermaterial und ihre Verschläge und Baulichkeiten für diese wissenschaftlichen Untersuchungen zur Verfügung stellte; des Dankes der Forscher kann sie sicher sein!

\section{TIERPSYCHOI,OGISCHE ARBEITEN, ZU WELCHEN DIE BEOB- ACHTUNGEN UND EXPERIMENTE AUSGEFÜHR'T WURDEN INNERHALB DES GARTENS DER KÖN. ZOOL. GES. „NATURA ARTIS MAGISTRA"}

I J. A. Bierens de HaAn. Die Balz des Argusfasans. Biol. Zentralbl., XLVI, 1926

2 - Versuche über das Sehen der Affen. IV. Das Erkennen gleichförmiger und ungleichförmiger Gegenstände bei niederen Affen. V. Erkennen Affen in zweidimensonalen Abbildungen ihnen bekannte Gegenstände wieder?

Zeitschr. für vergl. Physiol., V, 1927.

3 - Uber Wahl nach relativen und absoluten Merkmalen. (Versuche an Affen und Bienen).

Zeitschr. für vergl. Physiol., VII, 1928.

4 Uber das Suchen nach verstecktem Futter bei Affen und Halbaffen. Zugleich ein Beitrag zu der Frage nach dem konkreten Verständnis dieser Tiere. Zeitschr. für vergl. Physiol., XI, 1930.

5 - und Margaretha J. Frima, Versuche über den Farbensinn der Lemuren. Zeitschr. für vergl. Physiol., XII, 1930.

6 - Werkzeuggebrauch und Werkzeugherstellung bei einem niederen Affen (Cebus hypoleucus Humb.). Zeitschr. für vergl. Physiol., XIII, 193I.

7 - Uber das sogenannte "Waschen" des Waschbären (Procyon lotor), nebst einigen Bemerkungen über die Formen und die Bedeutung der tierischen Spiele. Biol. Zentralbl., LII, 1932.

8 - Uber das Suchen nach verstecktem Futter bei einigen Procyoniden und einem Eichhörnchen. Zeitschr. für vergl. Physiol., XVII, 1932.

9 - Der Stieglitz als Schöpfer. 'ourn. für Ornithologie, LXXXI, 1933.

10 - und J. Тн. Меукnecht. Dressurversuche an einem stark motorischen Affen. Biol. Zentralbl., LIV, 1934. 
II J. A. Bierens de HAan. Versuche über die Verwendung der Kiste als Schemel bei einigen Procyoniden (Wasch- und Nasenbären), nebst einigen Bemerkungen über das konkrete Verständnis der Tiere im allgemeinen.

Zeitschr. für Psychol., CXXXI, 1934.

12 Zahlbegriff und Handlungsrhythmus bei einem Affen.

Zool. Jahrb. Abt. allg. Zool. Physiol., LIV, 1935.

13 - und Dina Kooyman. Bildung und Zerstörung von Handlungsrhythmen bei einem Schweinsaffen. Arch. Néerl. Zool., II, 1936.

I4 - Versuche über das Erfassen räumlicher Beziehungen in waagerechter und senkrechter Richtung bei einigen niederen Affen. Zeitschr. für Tierpsychol., I, 1937.

i5 — Du degré d'intelligence chez les animaux et de leur place dans le système zoologique.

Journ. de Psycholog., XXXIV, 1937.

16 und Florrie Heubel. Uber Futtervorliebe bei Affen und die Bestimmung ihrer Grösse und Stärke. (Mit einem Versuch zu einer charakterologischen Deutung der Ergebnisse).

Zeitschr. für Morphol. und ర̌kol., XXXIV, ı.938.

17 Florrie Heubel. Beobachtungen und Versuche über das Sinnesleben und die Intelligenz eines Macropus giganteus Zimmermann. Bijdragen tot de Dierkunde, XXVII, 1938.

18 Monika Holzapfel. Uber Bewegungsstereotypien bei gehaltenen Säugern. I. Mitteilung. Bewegungsstereotypien bei Caniden und Hyaena. Zeitschr. für Tierpsychol., II, 1938.

$19-U$ Uber Bewegungsstereotypien bei gehaltenen Säugern. III. Mitteilung. Analyse der Bewegungs-stereotypie eines Gürteltieres (Dasypus villosus Desm.). Der Zool. Garten, X (im Druck).

19a. A. Kortlandt. De uitdrukkingsibewegingen en -geluiden van Phalacrocorax carbo sinensis (Shaw \& Nodder). Ardea, XXVII, 1938.

20 T. KUIPER. Een onderzoek naar het leervermogen van witte ratten. Meded. van de Ned. Stichting voor Psychot., I, 1935.

2I A. F. J. Poktrelje. Biologische opmerkingen uit de Diergaarde van het Kon. Zoologisch Genootschap „Natura Artis Migistra”. Bijdragen tot de Dierk., XXI, I919.

22 - Eenige merkwaardige instincten en gewoontevormingen bij vogels. Ardea, XI, 1922.

23 - Remarkable behaviour of the Whooperswan. Ardea, XII, 1923.

24 - Zur Ethologie bezw. Psychologie der Rhea americana L. Ardea, XIV, 1925.

25 - Zur Ethologie bezw. Psychologie von Botaurus stellarus (L.). Ardea, XV, 1926.

26 - Ornitho-psychologische opmerkingen. Ardea, XVI, 1927.

27 Zur Ethologie bezw. Psychologie von Phalacrocorax carbo subcormoranus (Brehm). Ardea, XVI, 1927.

28 Zur Ethologie bezw. Psychologie der Silbermöwe, Larus argentatus argentatus argentatus Pont. Ardea, XVII, 1928.

29 - Versuch zu einer verhaltungspsychologischen Deutung des Balzgebahrens der Kampfschnepfe Philomachus pugnax (L).

Proc. VIIth Intern. Ornithol. Congress Amsterdam 1930. 
30 A. F. J. Portielje. On a remarkable and purposive feeding behaviour in the seaanemone Diadumene cincta Stephenson, a short note on Metridium senile Var. pallidum (Holdsworth) and Anthopleura thallia (Gosse), also until now unknown on the Dutch shore, and a list of the Dutch Actiniaria.

Tijdschr. Ned. Dierk. Ver., III, I933.

3I — Ein bemerkenswerter Grenzfall von Polygamie bzw. accessorischer Promiskuität beim Höckerschwan, zugleich ein Beitrag zur Ethologie bezw. Psychologie von Cygnus olor (Gm.).

Journ. für Ornithol., LXXXIV, I936.

$32 \mathrm{~J}$. J. Sмiтh. Analyse der Wahrnehmung von Objekten mit mehreren Merkmalen (farbigen Objekten) bei Schweinsaffen.

Diss. Utrecht, 1938 (im Druck). 